\title{
Reflected Shock Tunnel Noise Measurement by Focused Differential Interferometry
}

\author{
N. J. Parziale* \\ California Institute of Technology, Pasadena, California, 91125, USA \\ J. E. Shepherd ${ }^{\dagger}$ H. G. Hornung ${ }^{\ddagger}$ \\ California Institute of Technology, Pasadena, California, 91125, USA
}

\begin{abstract}
A series of experiments is conducted where a quantitative non-intrusive optical technique is used to investigate disturbances in the free-stream of $T 5$, the free-piston driven reflected shock tunnel at Caltech. The optical technique, focused laser differential interferometry (FLDI), measures fluctuations in density. In the test matrix, reservoir enthalpy is varied while the reservoir pressure is held fixed. The results show the perturbations in density are not a strong function of the reservoir enthalpy. During one experiment, exceptional levels of noise were detected; this unique result is attributed to non-ideal operation of the shock tunnel. The data indicate that rms density fluctuations of less than $0.75 \%$ are achievable with attention to tunnel cleanliness. In addition, the spectral content of density fluctuation does not change throughout the test time.
\end{abstract}

\section{Nomenclature}

$h \quad$ Enthalpy, $(\mathrm{MJ} / \mathrm{kg})$

$P \quad$ Pressure, $(\mathrm{MPa})$

$U \quad$ Velocity, $(\mathrm{m} / \mathrm{s})$

$\rho \quad$ Density, $\left(\mathrm{kg} / \mathrm{m}^{3}\right)$

$I \quad$ Irradiance, $\left(\right.$ Watt $/ \mathrm{m}^{2}$ )

$K \quad$ Gladstone-Dale constant, $\left(\mathrm{m}^{3} / \mathrm{kg}\right)$

$L \quad$ Sensitive Optical Path Length (m)

$\lambda$ Wavelength (m)

$\Delta \phi \quad$ Phase Change (Radians)

$V \quad$ Potential (Volts)

$R$ Resistance (Ohms)

$f \quad$ Frequency $(\mathrm{Hz})$

$\mathcal{R}$ Responsivity (Amps/Watt)

$n$ Index of refraction

Subscript

$R \quad$ Reservoir Condition

$\infty \quad$ Free-stream Condition

\section{Introduction}

For nearly fifty years the study of boundary layer transition on hypersonic vehicles has been a topic of national and international research. Understanding transition is important in the design process of a

*PhD Candidate, Graduate Aeronautical Laboratories, 1200 E. California Blvd. MC 205-45, AIAA Student Member.

$\dagger$ Professor, Graduate Aeronautical Laboratories, 1200 E. California Blvd. MC 205-45, AIAA Member.

${ }^{\ddagger}$ Emeritus Professor, Graduate Aeronautical Laboratories, 1200 E. California Blvd. MC 205-45, AIAA Member. 
hypersonic vehicle because of the need to predict surface heating rates and skin friction coefficients, both of which may be several times higher when the boundary layer is turbulent. ${ }^{1}$ Several types of ground-test facilities are used in high-speed boundary layer transition research. One type is a reflected shock tunnel, an impulse facility capable of simulating hypervelocity flows with high reservoir enthalpy and high reservoir pressure, enabling the investigation of high-enthalpy effects on transition. The boundary layer receptivity problem makes the characterization of the noise environment in the free-stream a key aspect of studying transition in ground-test. ${ }^{2-5}$ An extensive review of the effect of tunnel noise on high-speed boundary layer transition has been made by Schneider; ${ }^{6}$ the major sources of disturbances in a shock tunnel are noted to be the turbulent boundary layer on the nozzle wall and/or the entropy fluctuations being advected from the reservoir of the facility.

Pitot pressure measurements are typically made to quantify the perturbations in the test gas. ${ }^{7}$ In a reflected shock tunnel this is difficult because: 1) The bandwidth of commercially available piezoelectric pressure transducers is too low $(<1 \mathrm{MHz})$ to cover the frequency band of importance. 2) The pressure transducers must be flush mounted to avoid resonances in any sort of protective cavity. ${ }^{8}$ Flush mounting the transducer causes excessive thermal loading and puts them at risk for particulate impact after the passage of the test gas. 3) It is uncertain that flush mounted pressure transducers in a Pitot probe configuration produce a faithful representation of the noise level in a supersonic or hypersonic free-stream. The interaction of free-stream fluctuations with the bow shock wave ${ }^{9}$ that forms in front of the transducer may thwart the goal of resolving a wide range of disturbance length scales. This interaction is known to be a function of the obliqueness $^{10}$ and the strength ${ }^{11}$ of the shock wave. Furthermore, the complexity of the subsonic flow-field behind the bow shock wave may further obscure the fidelity of free-stream disturbance measurement.

Non-intrusive optical methods are an alternative to direct mechanical measurement. One limitation to successful implementation is the harsh vibration environment in a large scale reflected shock tunnel, making proper alignment difficult to execute repeatedly. Another limitation is that many optical methods are line of sight integrating. They probe any media they traverse, including the turbulent shear layer that forms at the end of the nozzle wall; this adds irrelevant background noise to the measurement. Single point focused schlieren has been used to explore the noise levels in T5 with moderate success, primarily limited in effectiveness by the difficulty in calibrating the diagnostic. ${ }^{12}$ In another approach, the unsteadiness of the bow shock wave in front of a blunt body was measured in T5 by using an edge detection algorithm to track the position of the shock with high-speed schlieren. ${ }^{13}$

This paper describes a measurement technique and test results collected during an investigation of disturbances in the free-stream of T5. These experiments are part of a test campaign on hypervelocity boundary layer transition research currently being conducted in $\mathrm{T} 5 .{ }^{12,14-16}$ The quantitative non-intrusive optical technique, focused laser differential interferometry (FLDI), has been successfully implemented to make quantitative measurements of density perturbations of the free-stream at the nozzle exit with high temporal $(25 \mathrm{MHz})$ and spatial $(700 \mu \mathrm{m})$ resolution. The experimental setup, bench testing, and results are presented.

\section{Facility}

All measurements are made in T5, the free-piston driven reflected shock tunnel at the California Institute of Technology (Fig. 1). It is the fifth in a series of shock tunnels designed to simulate high-enthalpy effects on aerodynamics of vehicles flying at hypervelocity speeds in planetary atmospheres. More information regarding the capabilities of $\mathrm{T} 5$ can be found in the literature. ${ }^{17}$

An experiment and typical operating conditions are as follows: a $120 \mathrm{~kg}$ aluminum piston is loaded into the compression tube/secondary reservoir junction. A secondary diaphragm (mylar, $127 \mu \mathrm{m}$ thick) is inserted at the nozzle throat at the end of the shock tube near the test section and a primary diaphragm (stainless steel, $7 \mathrm{~mm}$ thick) is inserted at the compression tube/shock tube junction. The test section, shock tube and compression tube are sealed, then evacuated. The shock tube is filled with the test gas (in the present study, air) to $40-120 \mathrm{kPa}$, the compression tube is filled with a $\mathrm{He} / \mathrm{Ar}$ mixture to $100 \mathrm{kPa}$ and the secondary reservoir is filled with air to $10 \mathrm{MPa}$. The air in the secondary reservoir is released, driving the $120 \mathrm{~kg}$ aluminum piston into the compression tube. This piston motion compresses the driver gas to the rupture pressure of the primary diaphragm $(100 \mathrm{MPa})$. Following the primary diaphragm rupture, a shock wave propagates in the shock tube, is reflected off the end wall, breaking the secondary diaphragm and re-processing the test gas. The test gas is then at high temperature $(6000 \mathrm{~K})$ and pressure $(60 \mathrm{MPa})$ with negligible velocity, and is then expanded through a converging-diverging contoured nozzle to Mach 5.5 


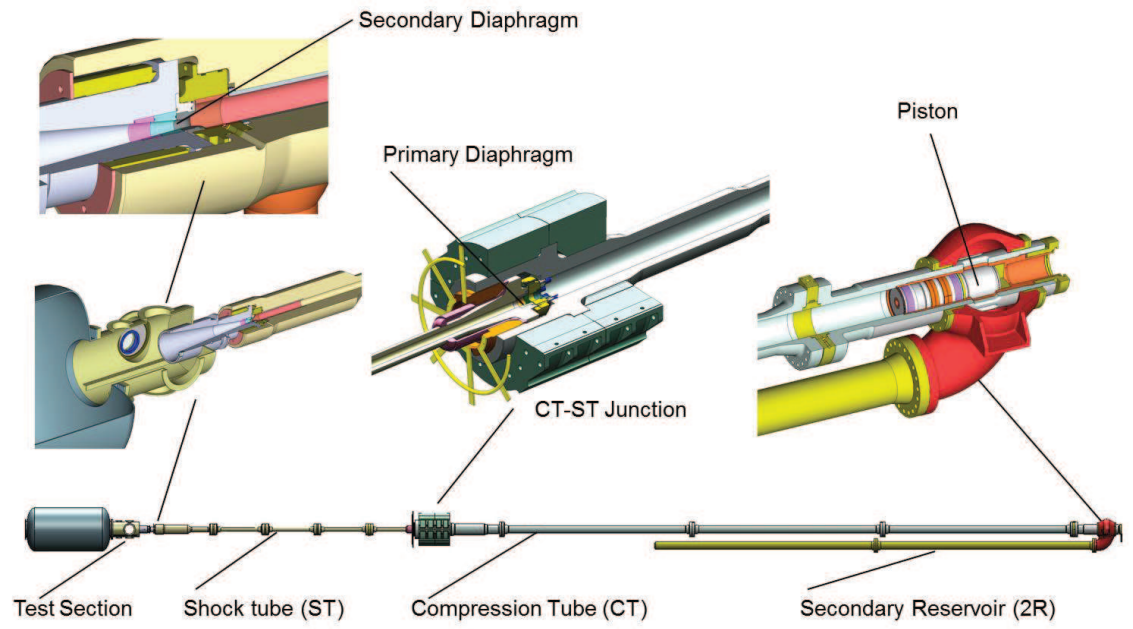

Figure 1. Above is a schematic of T5 with a blown up view of each of the major sections.

in the test section.

\section{Measurement Technique}

The measurement technique chosen for this study is a specific type of two beam interferometry, focused laser differential interferometry (FLDI). Smeets developed the technique, along with a number of similar configurations, at ISL (Saint Louis, France) for use in gas dynamic studies in the 1970's. ${ }^{18-21}$

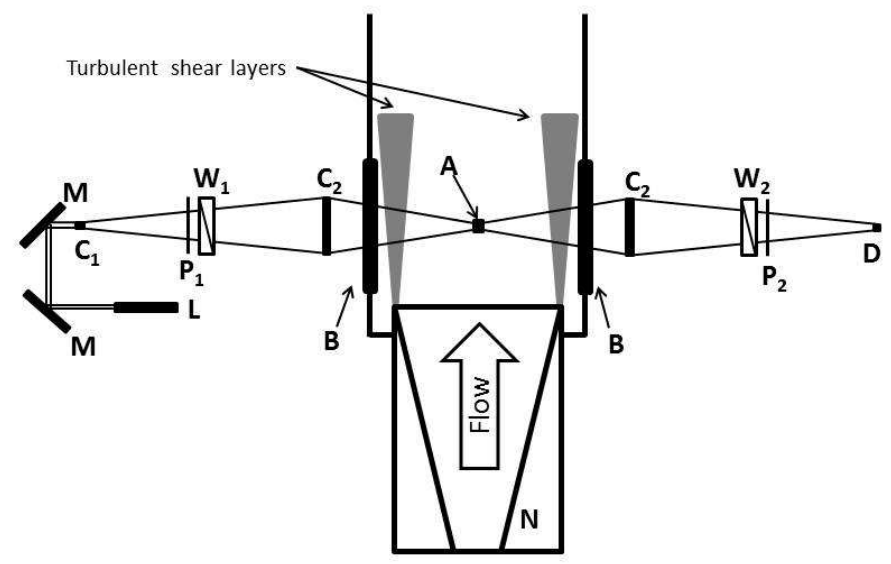

Figure 2. Above is an annotated schematic of the FLDI. L, Laser; M, mirror; C, lens; P, polarizer; W, Wollaston prism; B, window; A, probe volume; D, photodetector; N, nozzle.

Following the optical path in Fig. 2, starting from the laser (200 mW Spectra Physics Excelsior-532200-CDRH), the beam is turned by a periscope arrangement for precise directional control. The beam is expanded by a lens, $\mathrm{C}_{1}$, and linearly polarized by $\mathrm{P}_{1}$ at $45^{\circ}$ to the polarization axes of the first Wollaston prism, $\mathrm{W}_{1}$. The two polarization axes of $\mathrm{W}_{1}$ lie in the plane normal to the horizontal span-wise direction. The prism splits the light by a narrow angle (2 arc minutes) in the stream-wise direction into orthogonally 
polarized beams. One beam is polarized in the stream-wise direction, the other beam is polarized in the vertical span-wise direction. The separation of the beams is fixed at $350 \mu \mathrm{m}$ by a lens, $\mathrm{C}_{2}$, while the diameter of the beams is reduced to a point in the center of the test section. This arrangement creates two beams with orthogonal polarization that share much of the same optical path except within $\pm 15 \mathrm{~mm}$ of the focal point (along the beam direction, centered at A in Fig. 2, shown in detail in Fig. 3). In the focal region, the beams are less than $100 \mu \mathrm{m}$ in diameter, and traverse separate but very closely spaced volumes; they are $350 \mu \mathrm{m}$ apart, assuming $1 / e^{2}$ Gaussian beam propagation. ${ }^{22}$ It is primarily within this small focal region that the diagnostic is sensitive to changes in optical path length (OPL). Beyond the beam focus, the optical paths are again common and outside the test section an additional lens, $\mathrm{C}_{2}$, re-focuses the beams. The Wollaston prism, $\mathrm{W}_{2}$, and polarizer, $\mathrm{P}_{2}$, recombine and then mix the orthogonally polarized beams, such that the interference will be registered as irradiance fluctuations by the photodetector. The response of the photodetector (22.5V battery biased FDS100 photodiode) is amplified (SRS SR445) at a gain of 5 and digitized at $100 \mathrm{MHz}$ by a 14-bit Ethernet oscilloscope (Cleverscope CS328A-XSE).

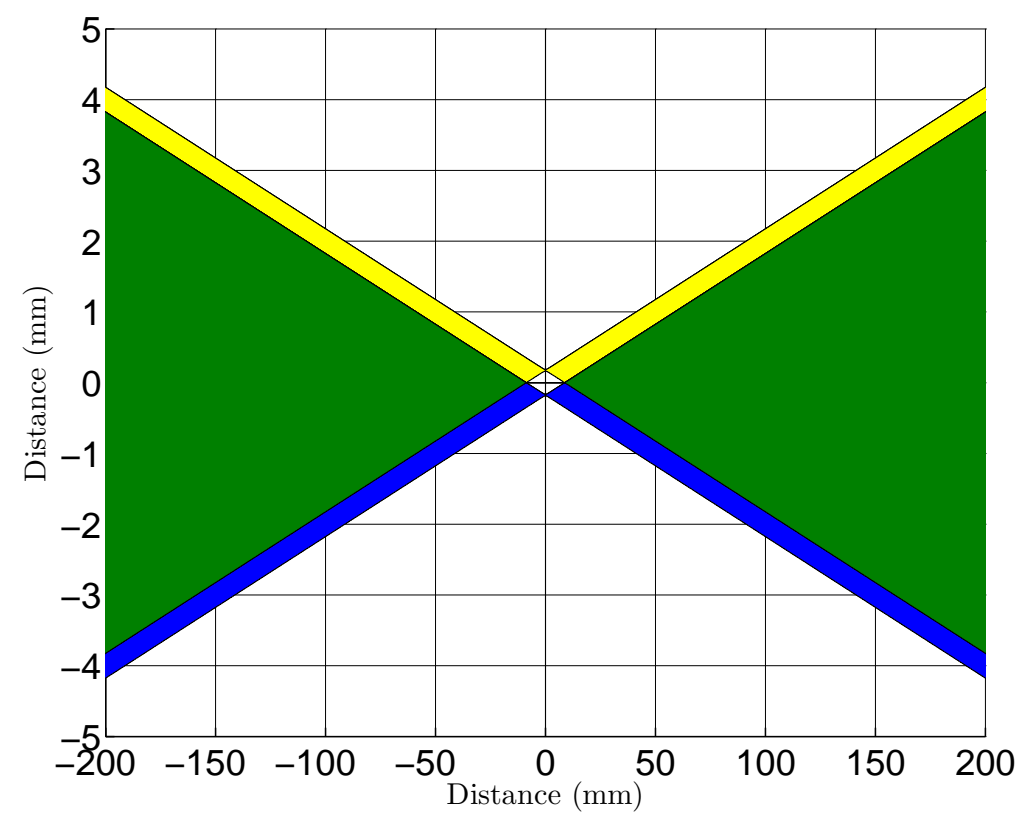

Figure 3. The calculated Gaussian profile shows that two orthogonally polarized beams (one in yellow, one in blue) have a common optical path (the common optical path is shaded in green) for the majority of the test section. The exception is at the focus, where the diagnostic is most sensitive.

A relation between the fluctuations in density and irradiance at the photodetector is found by considering the region within $\pm 15 \mathrm{~mm}$ of the focal point, along the beam direction, to be a two beam differential interferometer. The technique detects differences in phase, primarily due to the density differences at the two spatially separated focal regions; thus, making the interferometer sensitive to spatial density differences in the stream-wise direction. The relation between phase and change in irradiance due to change in OPL is

$$
I_{d}=I_{1}+I_{2}+2 \hat{l_{1}} \cdot \hat{l_{2}} \sqrt{I_{1} I_{2}} \cos (\Delta \phi),
$$

where $\Delta \phi$ is the phase change at the beam focus, $I_{d}$ is the irradiance at the detector's surface, and $I_{1}=I_{2}=I_{0}$ are the irradiances of the orthogonally polarized beams. After the beams are mixed by the second polarizer, their unit vectors' dot product, $\hat{l_{1}} \cdot \hat{l_{2}}$, is unity. The change in phase is

$$
\Delta \phi=\frac{2 \pi}{\lambda_{0}} \Delta O P L \approx \frac{2 \pi}{\lambda_{0}} L \Delta n
$$

where $L$ is the integration length over the phase object in the focal region, $\Delta n$ is the change in refractive index between the two beams, and $\lambda_{0}$ is the wavelength of the laser. From the Gladstone-Dale relationship,

$$
n=K \rho+1,
$$


Eq. 2 becomes

$$
\Delta \phi=\frac{2 \pi}{\lambda_{0}} L K\left(\rho_{\|}-\rho_{\perp}\right)=\frac{2 \pi}{\lambda_{0}} L K \Delta \rho
$$

The change in phase, $\Delta \phi$, is due to the difference in density, $\rho_{\|}-\rho_{\perp}=\Delta \rho$. The densities are the instantaneous local densities interrogated by the beams polarized parallel $\left(\rho_{\|}\right)$and orthogonal $\left(\rho_{\perp}\right)$ to the streamlines in the free-stream. The two beams are spaced $350 \mu \mathrm{m}$ apart, and the phase object is integrated over the OPL, $L$ (within $\pm 15 \mathrm{~mm}$ of the focal point). For comparison between experiments it is more convenient to think of density changes in non-dimensional terms. Normalizing $\Delta \rho$ by the mean local density, $\rho_{L}$ makes Eq. 4 ,

$$
\Delta \phi=\frac{2 \pi}{\lambda_{0}} L K \rho_{L} \frac{\Delta \rho}{\rho_{L}} .
$$

The potential response of the photodetector, $V$ is expressed as

$$
V=I \mathcal{R} R_{L}
$$

where $\mathcal{R}$ is the responsivity of the photodiode and $R_{L}$ is the load resistance. A relation for the normalized change in density in terms of the output voltage of the photodetector and fixed parameters of the apparatus is found by combining Eqs. 1, 5 and 6 as

$$
\frac{\Delta \rho}{\rho_{L}}=\frac{\lambda_{0}}{2 \pi K L \rho_{L}} \sin ^{-1}\left(\frac{V}{V_{0}}-1\right)
$$

The interferometer is set to the most linear part of a fringe before each experiment, so there is a $\pi / 2 \mathrm{rad}$ phase shift introduced, and $V_{0}=2 I_{0} \mathcal{R} R_{L}$. The phase shift, $\Delta \phi$ is less than $\pi / 3 \mathrm{rad}$, so there no fringe ambiguity.

\section{Bench Testing of Measurement Technique}

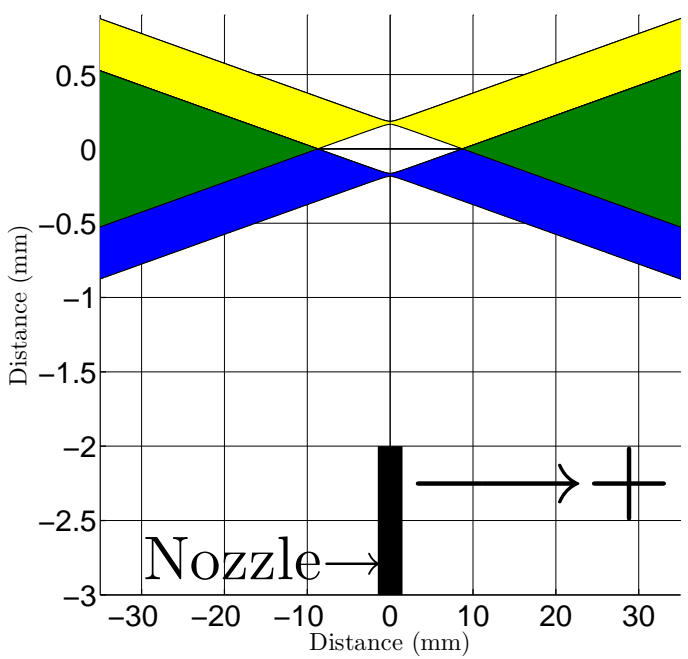

(a)

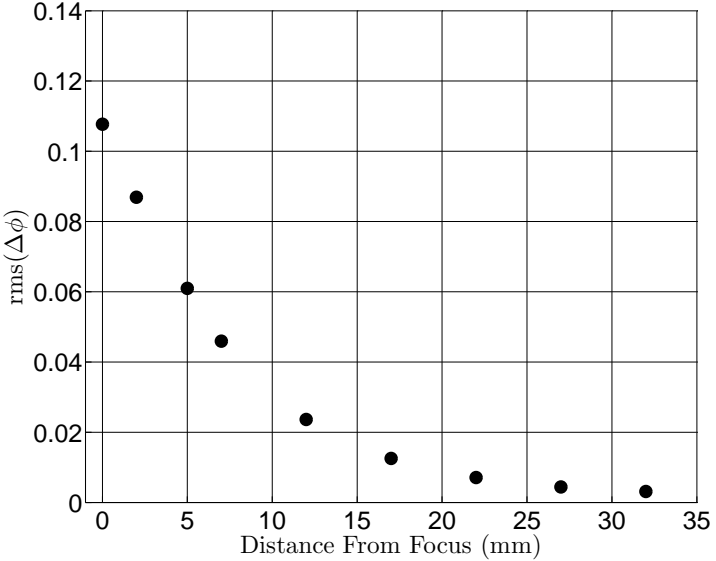

(b)

Figure 4. Above left (a), is a sketch of the small $\mathrm{CO}_{2}$ nozzle and laser beam profile. The arrow $(\rightarrow+)$ denotes the direction in which the nozzle is traversed. The yellow shaded area denotes where the laser is polarized in the stream-wise direction. The blue shaded area denotes where the laser is polarized normal to the page. The green shaded region denotes the area where the two laser beams overlap. Above right (b), are the results of the bench test; the results demonstrate the decrease in response of the FLDI with increasing distance from the sensitive region.

This section describes the experimental evaluation of the sensitive region of the FLDI. A subsonic turbulent $\mathrm{CO}_{2}$ jet is traversed in the horizontal span-wise direction along the direction of beam propagation (schematic in Fig. 4(a)). The metric used to evaluate the size and character of the sensitive region in the 
span-wise direction is the amplitude of fluctuation at the focus relative to the amplitude of fluctuation at a distance from the focus. The rms value of the phase function,

$$
r m s(\Delta \phi)=\sqrt{\frac{1}{N} \sum_{i=1}^{N}\left(\Delta \phi_{i}\right)^{2}},
$$

is used as the measure of fluctuation, where $N$ is the number of samples and $\Delta \phi$ (as in Eq. 5) in terms of the potentials $V$ and $V_{0}$, is,

$$
\Delta \phi=\sin ^{-1}\left(\frac{V}{V_{0}}-1\right) .
$$

The bench tests were conducted in the following manner: 1) Place the nozzle approximately 2 millimeters from the focus of the FLDI (this initial position is shown in Fig. 4(a)), 2) record data for 15 milliseconds, 3) translate the nozzle a small distance in the span-wise direction, 4) record data for 15 milliseconds, 5) repeat steps 3 and 4 until no noticeable perturbations in the oscilloscope reading are perceptible. The jet was confirmed to be subsonic by high-speed schlieren visualization.

The results of this bench test demonstrate the decrease in response of the FLDI with increase in distance from the focus (Fig. 4(b)). The $1 / e$ folding in rms response of the FLDI to a subsonic $\mathrm{CO}_{2}$ jet is $\approx 10 \mathrm{~mm}$ from the focus. The $1 / e^{2}$ folding in rms response of the FLDI is $\approx 15 \mathrm{~mm}$ from the focus. Double the $1 / e^{2}$ folding is chosen as the effective integration length, $L=30 \mathrm{~mm}$, for the FLDI (as in Eq. 2).

\section{Results and Discussion}

A test series using the measurement technique outlined in the previous section (see conditions in Table 1, computed by the codes ESTC ${ }^{23}$ and $\mathrm{NENZF}^{24}$ ) was executed to investigate the noise in the free-stream flow of T5. The test-matrix was designed to hold the reservoir pressure $\left(P_{R}\right)$ constant while varying the reservoir enthalpy $\left(h_{R}\right)$ through the useful range of conditions in T5.

Table 1. Below is a summary of parameters for this test series. The reservoir enthalpy, pressure and temperature are $h_{R}, P_{R}$, and $T_{R}$, respectively. The free-stream velocity, temperature, density, pressure, and unit Reynolds number are $U_{\infty}, T_{\infty}, \rho_{\infty}, P_{\infty}$, and $R e_{\infty}^{u n i t}$, respectively.

\begin{tabular}{ccccccccc} 
Shot & $\begin{array}{c}h_{R} \\
(\mathrm{MJ} / \mathrm{kg})\end{array}$ & $\begin{array}{c}P_{R} \\
(\mathrm{MPa})\end{array}$ & $\begin{array}{c}T_{R} \\
(\mathrm{~K})\end{array}$ & $\begin{array}{c}U_{\infty} \\
(\mathrm{m} / \mathrm{s})\end{array}$ & $\begin{array}{c}T_{\infty} \\
(\mathrm{K})\end{array}$ & $\begin{array}{c}\rho_{\infty} \\
\left(\mathrm{kg} / \mathrm{m}^{3}\right)\end{array}$ & $\begin{array}{c}P_{\infty} \\
(\mathrm{kPa})\end{array}$ & $\begin{array}{c}R e_{\infty}^{\text {unit }} \\
(1 / \mathrm{m})\end{array}$ \\
\hline 2684 & 8.32 & 46.5 & 5350 & 3680 & 1160 & 0.057 & 19.1 & $4.62 \mathrm{E}+06$ \\
2686 & 14.2 & 49.5 & 7630 & 4640 & 2090 & 0.039 & 24.7 & $2.64 \mathrm{E}+06$ \\
2687 & 16.2 & 49.3 & 8180 & 4910 & 2320 & 0.035 & 24.9 & $2.30 \mathrm{E}+06$ \\
2691 & 5.81 & 41.8 & 4220 & 3110 & 716 & 0.071 & 14.7 & $6.79 \mathrm{E}+06$ \\
2692 & 5.58 & 42.4 & 4100 & 3050 & 676 & 0.075 & 14.6 & $7.26 \mathrm{E}+06$ \\
2693 & 8.90 & 49.6 & 5610 & 3890 & 1280 & 0.059 & 21.9 & $4.61 \mathrm{E}+06$ \\
2694 & 18.0 & 49.9 & 8600 & 5150 & 2520 & 0.032 & 25.6 & $2.09 \mathrm{E}+06$
\end{tabular}

Seven high-pass filtered time traces of density perturbations (Fig. 5, top) illustrate that the noise level is similar through the range of reservoir enthalpy $\left(h_{R}\right)$, other than shot 2684 , which has a larger rms than the other experiments. This is explained by understanding the process of running T5. There are buffers that slow the piston to a stop after the primary diaphragm is ruptured (refer to Fig. 1), these buffers were destroyed during the prior experiment (shot 2683). The destroyed buffers, made from polyurethane, introduced material to the shock tube that was not completely removed during the normal cleaning process that is executed after each experiment. During the startup process of shot 2684, this buffer material was introduced to the flow, and registered as large fluctuations by the interferometer.

The spectral power-frequency plot (Fig. 5, middle) further illustrates the similarity for all tests other than shot 2684. The noise measured in the free-stream during shot 2684 appears to be of larger amplitude than other experiments at lower frequencies $(<200 \mathrm{kHz})$. Applying Taylor's hypothesis, we also present the data in a premulitplied spectral power-wavenumber plot (Fig. 5, bottom). The free-stream velocity (Table 1) 

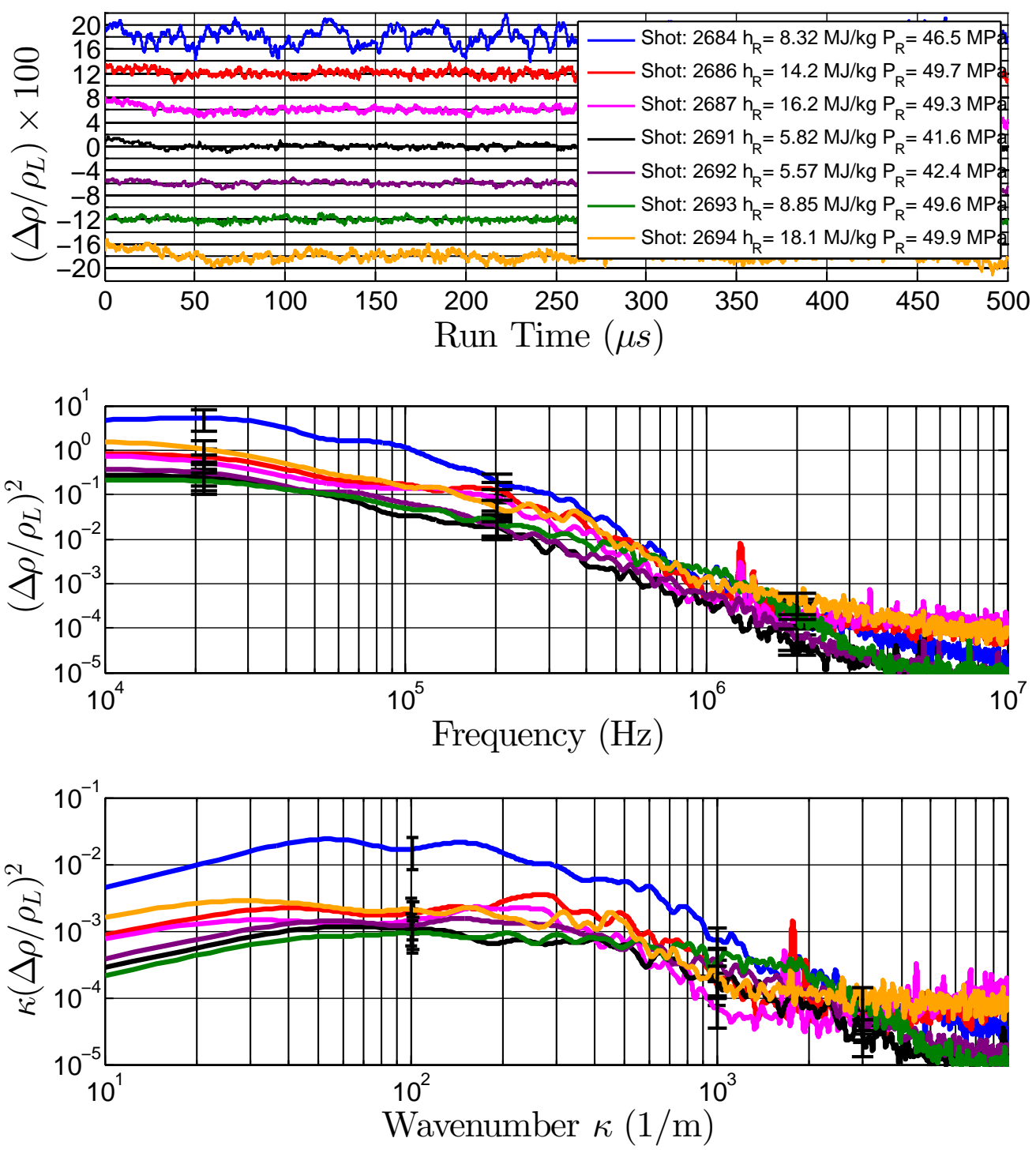

Figure 5. Above are filtered (filter cutoffs: $5 \mathrm{kHz}$ to $20 \mathrm{MHz}$ )time traces of $\Delta \rho / \rho \times 100$. Middle is a spectral power-frequency plot. Below is a premulitplied spectral power-wavenumber plot. The majority of the additional density perturbations at during shot 2684 are in the $10-200 \mathrm{kHz}$ band. The four RF spikes at $1.3,1.4$, 3.5 and $6 \mathrm{MHz}$ should be disregarded. These peaks appear when the tunnel is not in operation.

is used as the velocity scale. When plotted in this fashion, the spectra (other than shot 2684) appear to collapse within the uncertainty limits and there is a clear separation between shot 2684 and the other shots.

Characterizing the free-stream density disturbances is intended to aid high-speed boundary layer receptivity study research. The results presented in Fig. 5 are bandpass filtered at $5 \mathrm{kHz}$ to $20 \mathrm{MHz}$. Further interpretation of the results are given in Table 2. Rms density fluctuations are tabulated for each experiment for a range of cutoff frequencies. It is desirable to understand the amplitude of the rms density fluctuations at time scales of relavance to transition work being conducted in T5, so comparing the scaling of the most unstable frequency $\left(f_{M U}\right)$ in the boundary layer on a five degree half angle cone $\left(f_{M U} \approx 0.8 u_{e d g e} /\left(2 \delta_{99}\right)\right)^{25,26}$ to the measured frequency content of free-stream disturbance is a logical step. An estimate of the most amplified frequency is $f_{M U} \approx 1 \mathrm{MHz}$. The perspective of the most unstable frequency magnitude relative to the range of band pass cutoffs is important.

Examination of the spectral content evolution of fluctuations throughout the test time is important because there are complex non-steady wave systems in the reservoir of a reflected shock tunnel during the starting process and steady run time. The concern is that weak non-steady waves or driver gas contamina- 
Table 2. Below is a summary of rms density fluctuations at different bandpass filtered with different cutoffs, the quatity $\operatorname{rms}\left(\rho / \rho_{L}\right) \times 100$ is tabulated for each shot.

\begin{tabular}{cc|ccccccc}
$\begin{array}{c}\text { Cutoff } \\
\text { Low }\end{array}$ & High & 2684 & 2686 & 2687 & 2691 & 2692 & 2693 & 2694 \\
\hline $5 \mathrm{kHz}$ & $20 \mathrm{MHz}$ & 1.37 & 0.60 & 0.62 & 0.35 & 0.41 & 0.35 & 0.73 \\
$10 \mathrm{kHz}$ & $10 \mathrm{MHz}$ & 1.35 & 0.54 & 0.52 & 0.31 & 0.36 & 0.34 & 0.60 \\
$100 \mathrm{kHz}$ & $10 \mathrm{MHz}$ & 0.58 & 0.37 & 0.34 & 0.16 & 0.19 & 0.22 & 0.37 \\
$500 \mathrm{kHz}$ & $5 \mathrm{MHz}$ & 0.13 & 0.12 & 0.10 & 0.06 & 0.07 & 0.12 & 0.13
\end{tabular}

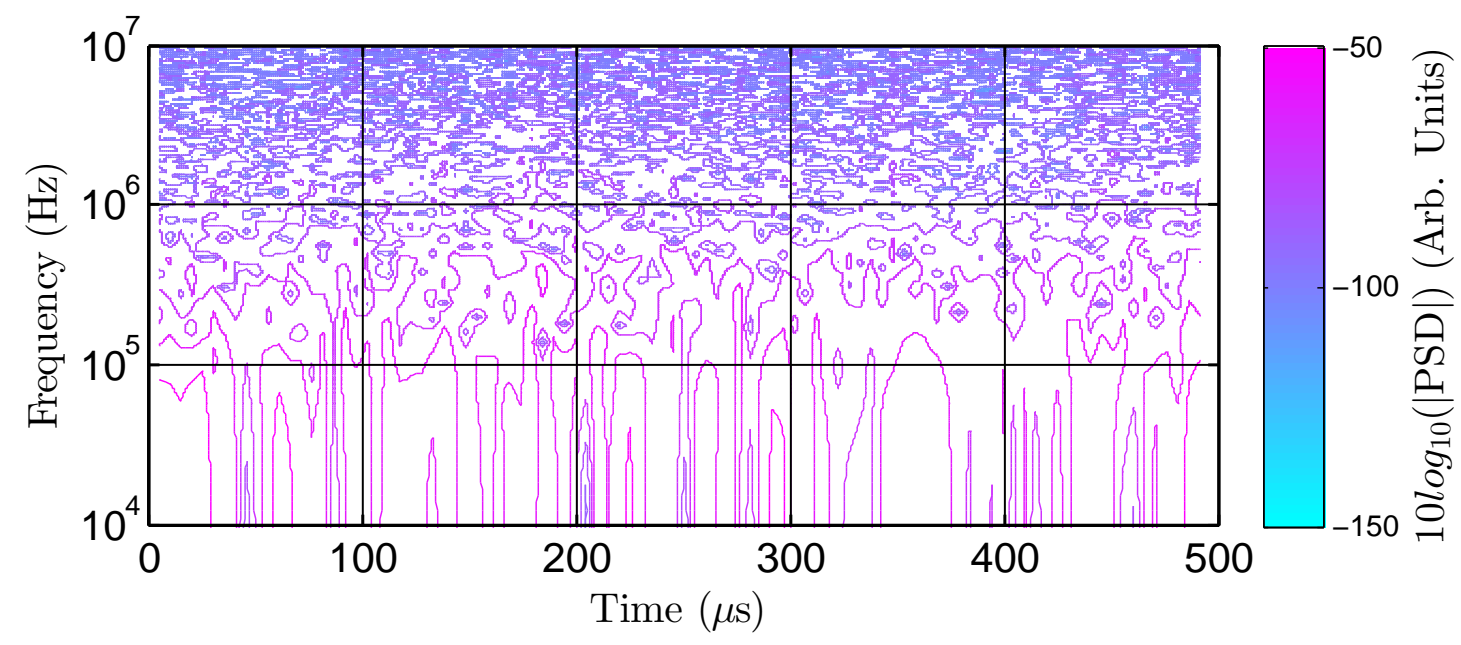

Figure 6. Above is a spectrogram (contours of power spectral density, plotted in time-frequency space) of shot $\mathbf{2 6 9 4}$, this illustrates how the spectral content of the fluctuations in the free-stream evolve throughout the test time. It is seen there are no clear trends in frequency-time space.

tion $^{27}$ may manifest themselves during the test time as trends in noise, i.e., the spectral content will shift because the driver gas (helium) leaks into the useful test flow. This point is addressed by constructing a spectrogram of the data presented in Fig. 5. A spectrogram is made by estimating the power spectral density (PSD) of $10.2 \mu$ s windows with a $50 \%$ overlap using the Welch method. An example (Fig. 6) shows contours of $10 \log _{10}(|\mathrm{PSD}|)$ in time-frequency space. The data indicate no clear trends in noise throughout the test time. The other experiments show a similar lack of trend in noise during the run time of the tunnel.

There are additional sources of noise that may vary with run condition, such as: 1) At higher reservoir enthalpy conditions for a fixed reservoir pressure, the Reynolds number in the nozzle is lower (because of higher viscosity and lower density). This may result in the boundary layer on the nozzle wall to transition to turbulence farther down-stream; the change in transition location on the nozzle wall could change the amount of noise the turbulent boundary layer contributes to the free-stream. 2) The contoured nozzle is being used at slightly off design conditions in this study. 3) For increasing reservoir enthalpy the contact discontinuity has a higher impedance mismatch. The higher the impedance mismatch, the lower the noise transmitted into the driven section from the driver section. Paul and Stalker ${ }^{28}$ studied this effect in expansion tubes. These effects on free-stream noise are difficult to quantify with the current technique, and are secondary compared to the need to operate the shock tube and nozzle in the cleanest possible manner.

\section{Uncertainty Estimate}

The systematic error stemming from applying Eq. 7 to the raw data is found by considering the propagation of uncertainty in $\Delta \rho / \rho_{L}$ as a function of the input parameters. ${ }^{29,30}$ The largest sources of systematic error are considered to be the uncertainty introduced by the assumed integration length, $L$ in Eq. 2 (assumed to be $30 \%$ ), the error in the voltages relative to the infinite fringe arrangement, $V$ and $V_{0}$ (assumed 
to be $50 \mathrm{mV}$ ), and the magnitude of the local density $\rho_{L}$ (assumed to be $20 \%$ ). This leads to an error of approximately $35 \%$ in the magnitude of $\Delta \rho / \rho_{L}$, with a $95 \%$ confidence interval. There is systematic error in the magnitude of $\Delta \rho / \rho_{L}$ from the spectral content estimation in each of the segments, this is approximately $20 \%$ in the magnitude of $\Delta \rho / \rho_{L}$, with a $95 \%$ confidence interval. Combining the errors from processing the data and estimating their spectra in a root-mean-squared sense, the systematic error is bounded at $40 \%$ (95\% confidence interval). This uncertainty is presented in the spectral content plots as error bars (Fig. 5).

Random error from electrical noise and mechanical vibrations can be estimated by inspecting the spectral content of the signal immediately preceding the test time. Approximately $10 \mathrm{~ms}$ before the test begins, vibration from the piston launch to compress the driver gas is transmitted through the steel rails that the entire shock tunnel rests on. By applying the identical signal processing scheme to the time just before the test, as used during the test, errors from ambient electrical noise and facility vibration can be bounded. There are peaks in the spectral content at 1.3, 1.4, 3.5 and $6 \mathrm{MHz}$. These peaks are from local radio stations and other unshielded electrical equipment. Precautions have been taken to eliminate such RF spikes in subsequent work. In the $5 \mathrm{kHz}$ to $20 \mathrm{MHz}$ frequency band, the spectral content from vibration and electrical noise is less than $0.5 \%$ in the magnitude of $\Delta \rho / \rho_{L}$ (95\% confidence interval); thus, the noise from mechanical vibration and electrical noise can be disregarded, except when interpreting the spectral plots at the noted frequencies.

\section{Conclusion}

A focusing laser differential interferometer has been constructed and implemented in T5. Free-stream noise levels were measured over a range of reservoir enthalpy, keeping the reservoir pressure fixed. The rms levels of free-stream fluctuations in density are quite similar through the range of reservoir enthalpy explored, except for one experiment. The larger rms response in the exceptional experiment can be attributed to an event in the tunnel on the prior experiment. The present results indicate that rms density fluctuations of less than $0.75 \%$ are achievable with attention to tunnel cleanliness. The data indicate no clear trends in the spectral content of density fluctuation throughout the test time. In addition, the frequency content at above $800 \mathrm{kHz}$ is of considerably smaller magnitude than the levels below $800 \mathrm{kHz}$, indicating that measurements of the acoustic instability ${ }^{31}$ (linear density disturbances) in hypervelocity boundary layers can be carried out in $\mathrm{T} 5$.

\section{Acknowledgments}

Thanks to Bahram Valiferdowsi for the isometric views of the solid model of the facility. Also, thanks to Joe Jewell for helping to compute the run conditions. This work was sponsored by AFOSR/National Center for Hypersonic Research in Laminar-Turbulent Transition, for which Dr. John Schmisseur and Dr. Deepak Bose are the program managers. The views and conclusions contained herein are those of the authors and should not be interpreted as necessarily representing the official policies or endorsements, either expressed or implied, of the Air Force Office of Scientific Research or the U.S. Government.

\section{References}

\footnotetext{
${ }^{1}$ Lin, T. C., "Influence of Laminar Boundary-layer Transition on Entry Vehicle Designs," Journal of Spacecraft and Rockets, Vol. 45, No. 2, 2008, pp. 165-175.

${ }^{2}$ Morkovin, M. V., "Critical Evaluation of Transition From Laminar to Turbulent Shear Layers with Emphasis on Hypersonically Traveling Bodies," AFFDL TR-68-149, 1969.

${ }^{3}$ Reshotko, E., "Boundary-Layer Stability and Transition," Annual Review of Fluid Mechanics, Vol. 8, 1976, pp. 311-349.

${ }^{4}$ Saric, W. S., Reed, H. L., and Kerschen, E. J., "Boundary Layer Receptivity to Freestream Disturbances," Annual Review of Fluid Mechanics, Vol. 34, 2002, pp. 291-319.

${ }^{5}$ Fedorov, A., "Receptivity of a High-Speed Boundary Layer to Acoustic Disturbances," Journal of Fluid Mechanics, Vol. 491, 2003, pp. 101-129.

${ }^{6}$ Schneider, S. P., "Effects of High-Speed Tunnel Noise on Laminar-Turbulent Transition," Journal of Spacecraft and Rockets, Vol. 38, No. 3, 2001, pp. 323-333.

${ }^{7}$ Bounitch, A., Lewis, D. R., and Lafferty, J. F., "Improved Measurements of Tunnel Noise Pressure Fluctuations in the AEDC Hypervelocity Wind Tunnel No. 9," Proceedings of 49th AIAA Aerospace Sciences Meeting Including the New Horizons Forum and Aerospace Exposition, AIAA, Orlando, Florida, 2011.

${ }^{8}$ McGilvray, M., Jacobs, P. A., Morgan, R. G., Gollan, R. J., and Jacobs, C. M., "Helmholtz Resonance of Pitot Pressure
} 
Measurements in Impulsive Hypersonic Test Facilities," AIAA, Vol. 47, No. 10, October 2009, pp. 2430-2439.

${ }^{9}$ Maheash, K., Lee, S., Lele, S., and Moin, P., "The Interaction of an Isotropic Field of Acoustic Waves with a Shock Wave," Journal of Fluid Mechanics, Vol. 300, 1995, pp. 383-407.

${ }^{10}$ Moore, F. K., "Unsteady Oblique Interaction of a Shock Wave with a Plane Disturbance," NACA TR-1165, 1954.

${ }^{11}$ Lee, S. S., Lele, S., and Moin, P., "Interaction of Isotropic Turbulence with Shock Waves: Effect of Shock Strength," Journal of Fluid Mechanics, Vol. 340, 1997, pp. 225-247.

${ }^{12}$ Parziale, N. J., Jewell, J. S., Shepherd, J. E., and Hornung, H. G., "Shock Tunnel Noise Measurement with Resonantly Enhanced Focused Schlieren Deflectometry," Proceedings of the 28th International Symposium on Shock Waves, ISSW, Manchester, UK, 2011.

${ }^{13}$ Marineau, E. C. and Hornung, H. G., "Study of Bow-Shock Wave Unsteadiness in Hypervelocity Flow from Reservoir Fluctuations," Proceedings of 48th AIAA Aerospace Sciences Meeting Including the New Horizons Forum and Aerospace Exposition, AIAA, Orlando, Florida, 2010.

${ }^{14}$ Jewell, J. S., Leyva, I. A., Parziale, N. J., and Shepherd, J. E., "Effect of Gas Injection on Transition in Hypervelocity Boundary Layers," Proceedings of the 28th International Symposium on Shock Waves, ISSW, Manchester, UK, 2011.

${ }^{15}$ Parziale, N. J., Jewell, J. S., Shepherd, J. E., and Hornung, H. G., "Optical Detection of Transitional Phenomena on Slender Bodies in Hypervelocity Flow," Proceedings of AVT-200 Specialists' Meeting on Hypersonic Laminar-Turbulent Transition, NATO, San Diego, California, 2012.

${ }^{16}$ Jewell, J. S., Parziale, N. J., Leyva, I. A., Shepherd, J. E., and Hornung, H. G., "Turbulent Spot Observations within a Hypervelocity Boundary Layer on a 5-degree Half-Angle Cone," Proceedings of 42nd AIAA Fluid Dynamics Conference and Exhibit, AIAA, New Orleans, Louisiana, 2012.

${ }^{17}$ Hornung, H. G., "Performance Data of the New Free-Piston Shock Tunnel at GALCIT," Proceedings of 17th AIAA Aerospace Ground Testing Conference, AIAA, Nashville, TN, 1992.

${ }^{18}$ Smeets, G. and George, A., "Gas Dynamic Investigations in a Shock Tube using a Highly Sensitive Interferometer," Translation of isl internal report 14/71, Original 1971, Translation 1996.

${ }^{19}$ Smeets, G., "Laser Interferometer for High Sensitivity Measurements on Transient Phase Objects," IEEE Transactions on Aerospace and Electronic Systems, Vol. AES-8, No. 2, March 1972, pp. 186-190.

${ }^{20}$ Smeets, G. and George, A., "Laser Differential Interferometer Applications in Gas Dynamics," Translation of ISL Internal Report 28/73, Original 1975, Translation 1996.

${ }^{21}$ Smeets, G., "Flow Diagnostics by Laser Interferometry," IEEE Transactions on Aerospace and Electronic Systems, Vol. AES-13, No. 2, March 1977, pp. 82-90.

${ }^{22}$ Siegman, A. E., Lasers, University Science Books, 1986.

${ }^{23}$ Mcintosh, M. K., "A Computer Program for the Numerical Calculation of Equilibrium and Perfect Gas Cnditions in Shock Tunnels," Technical Report CPD 169, Australian Defense Scientific Service, 1969.

${ }^{24}$ Lordi, J., Mates, R. E., and Moselle, J. R., "Computer Program for the Numerical Solution of Nonequilibrium Expansions of Reacting Gas Mixtures," NASA CR-472, 1966.

${ }^{25}$ Stetson, K. and Kimmel, R. L., "On Hypersonic Boundary-Layer Stability," Proceedings of the 13th Aerospace Sciences Meeting and Exhibit, AIAA, Reno, NV, 1992.

${ }^{26}$ Federov, A., "Transition and Stability of High-Speed Boundary Layers," Annual Review of Fluid Mechanics, Vol. 43, August 2011, pp. 79-95.

${ }^{27}$ Sudani, N. and Hornung, H. G., "Gasdynamical Detectors of Driver Gas Contamination in a High-Enthalpy Shock Tunnel," AIAA, Vol. 36, No. 3, March 1998, pp. 313-319.

${ }^{28}$ Paul, A. and Stalker, R. J., "Test Flow Disturbances in an Expansion Tube," Journal of Fluid Mechanics, Vol. 245, 1992, pp. $493-521$.

${ }^{29}$ Kline, S. J. and McClintock, F. A., "Describing Uncertainties in Single Sample Experiments," Mechanical Engineering, Vol. 75, 1953, pp. 3-8.

${ }^{30}$ Beckwith, T. G., Marangoni, R. D., and Lienhard, J. H., Mechanical Measurments, Pearson-Prentice Hall, Sixth ed., 2007.

${ }^{31}$ Mack, L. M., "Linear Stability Theory and the Problem of Supersonic Boundary-layer Transition," AIAA, Vol. 13, No. 3, July 1975 , pp. 278-289. 